\title{
Anesthetic management for neonate with giant cystic hygroma involved upper airway -A case report-
}

\author{
Hyunzu Kim ${ }^{1}$, Hyuk-Soo Kim ${ }^{1}$, Jung-Tak $\mathrm{Oh}^{2}$, and Jeong Rim Lee ${ }^{1}$ \\ Departments of ${ }^{1}$ Anesthesiology and Pain Medicine, ${ }^{2}$ Surgery, Yonsei University College of Medicine, Seoul, Korea
}

\begin{abstract}
Significant differences exist between neonatal and adult airways. Anesthetic management of the airway may be challenging in neonate and young infant with large neck mass because these patients are at risk for sudden complete airway occlusion resulting in hypoventilation and hypoxemia. We experienced a 30-day-old baby presented with large cystic hygroma on the left side of neck. This mass was infiltrated in pharynx and large enough to disturb swallowing and breathing, and was not reduced despite of sclero-therapy. Therefore he was decided to get surgical removal. During the gaseous induction with sevoflurane, spontaneous respiration was maintained because difficulty was encountered with intubation. Intraoperatively, the endotracheal tube was dislodged unexpectedly because vigorous surgical traction. Postoperatively the baby was extubated 2 day after operation, and suffered from transient facial nerve palsy and continuous discharge from surgical wound. He was administered ICU for a long time. (Korean
\end{abstract} J Anesthesiol 2011; 60: 209-213)

Key Words: Cystic hygroma, Difficult airway, Neonate.

For anesthesia of a patient with a giant lump that requires anesthesia and surgery, an anesthesiologist should be prepared for various circumstances that may arise with respect to management of the respiratory tract. Even an anatomically normal respiratory tract is susceptible to upper airway obstruction and respiratory distress and hypoxia can easily take place. Muscle composition, thoracic cage structure, functional residual capacity and oxygen consumption [1] must be taken into account, especially in newborn and young infants who require more care [2]. Thus, management of the respiratory tract of a newborn infant with a giant cervical lump may be a very difficult task. One common disease in newborn infants that may bring about such a situation is cystic hygroma. Cystic hygroma is a transilluminating, painless, soft and benign tumor composed of various sizes of cystic lumps [3]. Cystic hygroma is known to be a developmental malformation congenitally originating from the vascular lymphatic system and has a genetic association. The prevalence rate is one out of 6,000 to

Received: September 1, 2010. Revised: October 13, 2010. Accepted: October 30, 2010.

Corresponding author: Jeong Rim Lee, M.D., Ph.D., Department of Anesthesiology and Pain Medicine, Yonsei University College of Medicine, 134, Shinchon-dong, Seodaemun-gu, Seoul 120-752, Korea. Tel: 82-2-2228-2420, Fax: 82-2-364-2951, E-mail: MANYA@yuhs.ac

(c) This is an open-access article distributed under the terms of the Creative Commons Attribution Non-Commercial License (http:// creativecommons.org/licenses/by-nc/3.0/), which permits unrestricted non-commercial use, distribution, and reproduction in any medium, provided the original work is properly cited. 
16,000 newborn infants in the US [4]. However, only about 40$50 \%$ are identified in infants, but $90 \%$ are identified by the age of $2[3]$.

Since cystic hygroma is a disease of the lymphatic system, it can arise anywhere in the body; however, $70-80 \%$ of cases arise in the neck, particularly in the left posterior cervical triangle [5]. Cystic hygroma at the cervical region may accompany tracheal deviation or upper airway obstruction depending on the degree of invasion to the tongue, oral cavity, hypopharynx and larynx; thus, anesthesiologists may have difficulty in securing and maintaining the airway. Further, additional difficulty may arise in maintaining the airway during anesthesia of newborn infants because awake intubation using bronchoscopy is not easy, and unintended extubation is highly probable during the surgery due to short bronchus length. Moreover, complications and extubation time also need to be considered depending on the invasiveness of the surgery.

We investigated anesthesia management of a 30-day-old infant who underwent surgical removal of cystic hygroma that had invaded the upper airway, in terms of safe and effective securing and maintaining of the airway based on our experience in anesthesia and postoperative treatment procedures.

\section{Case Report}

The patient was diagnosed with suspected cystic hygroma, as a $5.8 \mathrm{~cm}$-sized left cervical lump was found on the prenatal ultrasonography performed before birth on the third day of the 22nd week of intrauterine pregnancy. Since the Quad test result was 1.74, suspecting Down syndrome, a gene test was carried out resulting in 46, XX. Assuming that normal vaginal

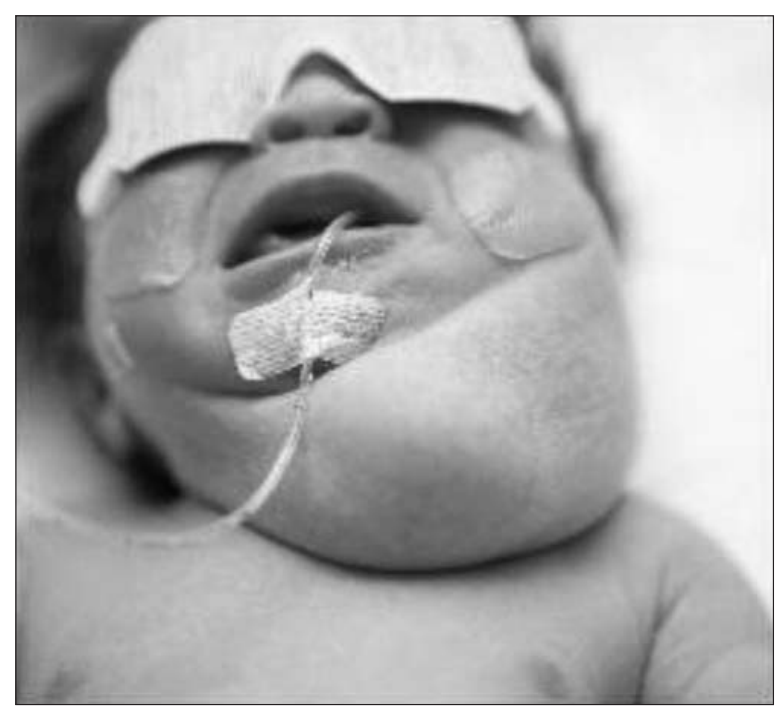

Fig. 1. Huge movable mass is found out on the left neck. delivery would be difficult due to the cervical lump, cesarean section was performed on the fifth day of the 38th week of intrauterine pregnancy. Weight of the infant was $3.3 \mathrm{~kg}$ at the time of birth, and he was transported to the intensive care unit for newborn infants right after delivery (Fig. 1). Cervical ultrasonography performed on the 1st day after birth revealed a multiseptate and cystic lump, extending from the anterior midline of the left cervix to the posterior midline in the horizontal direction and from the lower jaw to the mediastinum in the vertical direction. Cervical CT (computed tomography) showed that the lump extended from the skull base to the superior mediastinum, pressing the left internal auditory canal, surrounding the left carotid artery and jugular vein, and was connected to the sublingual gland and bottom of the hyoid bone. The trachea and thyroid gland were pushed to the right (Fig. 2) and hemorrhage in the lump was suspected because a

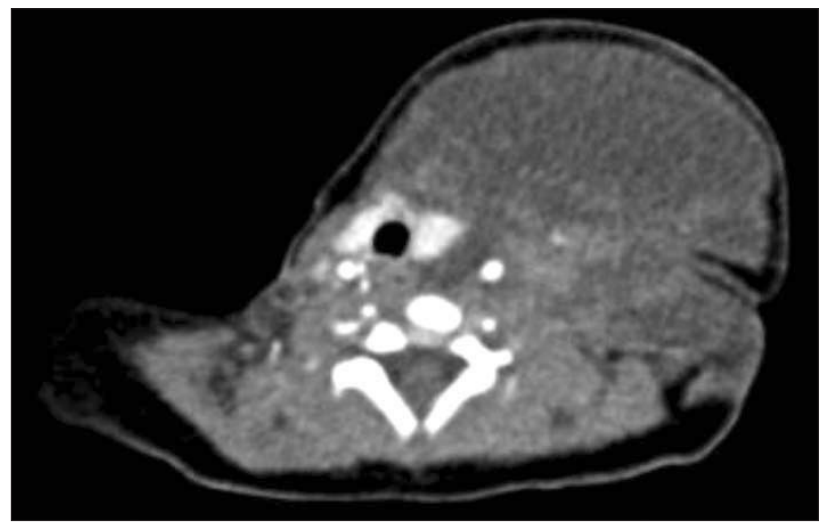

Fig. 2. Neck computed tomography shows cystic hygroma in the left neck, trachea and thyroid gland deviated to right side.

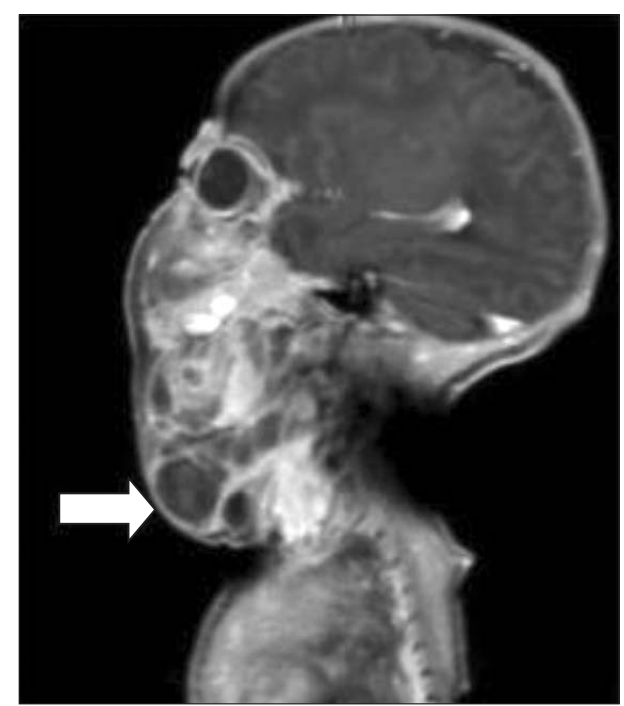

Fig. 3. Neck magnetic resonance imaging shows hemorrhagic lesion in cystic hygroma mass (arrow). 
thick septum was found and the precontrast test showed high attenuation (Fig. 3). After suction of the lump on the 14th day after birth, picibanil, the curing agent, was injected but the size of the lump was not significantly reduced. Hence, surgery was planned and the patient was moved to the operation room on the 30th day after birth for removal of the cervical cystic hygroma. Preop body weight was $5.4 \mathrm{~kg}$, and the size of the cervical lump was about $1 / 5$ of the patient's height. Arriving at the operation room, noninvasive blood pressure monitoring, electrocardiography and pulse oxymetry were installed, and respiration was assisted by using a facemask and setting the fresh gas flow at $6 \mathrm{~L} / \mathrm{min}$ and oxygen concentration at $100 \%$, while maintaining spontaneous ventilation. Jaw thrust was not easy because of the cervical lump. Sevoflurane concentration was gradually increased until the inspiratory concentration became $6 \%$. Meanwhile, fentanyl $1 \mathrm{mcg} / \mathrm{kg}$ was intravenously injected twice (half-does each time). Following approximately 10 minutes of ventilation assistance, it was decided that an appropriate level of anesthesia was reached, as spontaneous ventilation was still maintained; thus a laryngoscope was inserted for intubation. However, the epiglottis was not in the normal position, and, meanwhile, spontaneous ventilation was lost and oxygen saturation decreased. After increasing oxygen saturation by assisting ventilation with the facemask, the laryngoscope was inserted again. Verifying that cervical anatomical structures were lateralized to the right because of the lump and epiglottis was located not in the 12 o'clock direction but to the 2 o'clock direction, endotracheal intubation was carried out using a general uncuffed endotracheal tube of $3.0 \mathrm{~mm}$ inner diameter. After intubation, endotracheal tube depth was checked by auscultation and was found to exceed the carina when depth from the lips was greater than $11 \mathrm{~cm}$. The tube was then fixed at the tip of the right lip so that the depth would be $10 \mathrm{~cm}$. Intravenous injection of rocuronium $3 \mathrm{mg}$ followed. Ventilation per one time was set at $50 \mathrm{ml}$, and the corresponding peak airway pressure was measured at 28$30 \mathrm{cmH}_{2} \mathrm{O}$. When maintaining anesthesia, end-tidal $\mathrm{CO}_{2}$ was kept at $34-38 \mathrm{mmHg}$, systolic blood pressure at $50-66 \mathrm{mmHg}$, diastolic blood pressure at $18-34 \mathrm{mmHg}$, heart rate at $125-$ 160 beats per minute, body temperature at $35.4-36.7^{\circ} \mathrm{C}$ and peripheral oxygen saturation at $100 \% .2-3 \%$ of sevoflurane was used for the anesthesia maintenance, and rocuronium $1 \mathrm{mg}$ was intravenously injected for muscle relaxation if necessary. The surgeon inserted a venous catheter preop through the right internal jugular vein, and fluid was maintained using Hartmann's solution and normal saline through the catheter.

At 5 hours and 55 minutes after initiation of the operation, airway pressure suddenly increased up to $40 \mathrm{cmH}_{2} \mathrm{O}$ and tidal volume and end-tidal $\mathrm{CO}_{2}$ graphs were lost during traction of the lump. Endotracheal tube depth was fixed at $10 \mathrm{~cm}$ from the lips, as at the time of anesthesia induction. Suspecting obstruction of the endotracheal tube by discharged material, endotracheal tube suction was tried, but almost no discharge was found. Hence, the operation was stopped, the surgical drape was removed, and the laryngoscope was inserted. It was found that the tip of the endotracheal tube was protruding into the upper part of the vocal cord. Endotracheal intubation was carried out once again, and depth was fixed at $11 \mathrm{~cm}, 1 \mathrm{~cm}$ deeper than in the first trial. Meanwhile, peripheral oxygen saturation was reduced to $70 \%$ and then recovered to $100 \%$ after endotracheal intubation. Immediately after re-intubation, dexamethasone $1 \mathrm{mg}$ was intravenously injected.

Total duration of the operation was 7 hours and 30 minutes, total amount of crystalloid solution injected during the operation was $410 \mathrm{ml}$, discharged amount of urine was $25 \mathrm{ml}$, and estimated blood loss was $40 \mathrm{ml}$. Finishing the operation, fentanyl $10 \mathrm{mcg}$ was intravenously injected and the patient was moved to the intensive care unit for newborn infants under sedation and Ambu bagging.

Extubation was carried out on the second day postop, and no complication in the upper airway was found immediately after extubation. However, from the sixth day post-op on, facial expression asymmetry and left paralysis were found, which spontaneously improved on the 14th day postop. Continuous hemorrhaging of the operated parts until the 26th day in the intensive care unit occurred; thus, packed RBC transfusion was performed 3 times. On ultrasonography (33rd day postop), cystic tissue remained from the left inferior parotid gland to the posterior cervix, and a part of it was extended over the midline to the right cervix. Thus, additional picibanil was injected. The infant was given conservative treatment and discharged on the 47th day postop.

\section{Discussion}

Cystic hygroma is a benign tumor without potential malignancy. Applicable treatments include continued observation, repetitive suction, injection of curing agent into the lump, radiation therapy and radio frequency treatment. However, the only ultimate treatment method is surgical removal [6] and the general time for surgery is known to be 18 months to 2 years of age [7]. However, when the size of the lump is very large, a newborn infant may need surgery because it can cause respiratory distress [8] or swallowing difficulty [7]. In our case, since the size was large, spontaneous effacement was hardly expected, and the surgeon preferentially tried suction and curing agent injection considering the aesthetic difficulty; however, cyst size was not effectively reduced. Eventually, there was no choice but surgical treatment. In addition, care should be taken even in cases with small-sized cystic hygroma because 
the size can suddenly increase by infection, inflammation or internal hemorrhage. Therefore, emergency operation may be needed due to sudden respiratory arrest, hypovolemic shock and sepsis [7].

For an infant patient needing removal of cystic hygroma, it is most important for the anesthetist to understand the range of invasion into the respiratory tract by the lump and prepare for airway management. Based on our case, it is understood that airway management should consider all procedures before and after anesthesia, especially difficulties in induction and intubation caused by anatomical changes, endotracheal tube management during anesthesia, determination of the time for postoperative extubation and complications of the respiratory organs.

The first consideration is securing the airway during anesthesia. Newborn infants are difficult to intubate, and, consequently, the success ratio of intubation in this group is only 60. In addition, because newborn infants may rapidly move to a state of hypoxia when trying intubation, it is recommended to limit intubation duration to less than 20 seconds. However, according to the study mentioned above, a median of 33 seconds was taken, and an average of 28 seconds was taken by specialists whose career was longer than 5 years [9]. Therefore, more preparation is required for infants with anatomical changes in the respiratory tract because of the cervical lump, but there are few methods for anesthetists to choose from. First, if the patient is an adult, awake endotracheal intubation using fibroptic bronchoscopy may be preferentially considered in a similar case. However, cooperation from an infant is almost impossible during this method, so awake intubation is rarely chosen. Eventually, intubation should be carried out under general anesthesia or deep sedation, and spontaneous ventilation must be maintained until intubation success during the procedure [10]. It is known that inhalation anesthetic is preferred by many anesthetists and is favored more as the age of the infant decreases [11]. Therefore, in this case, sevoflurane was gradually inhaled to induce loss of consciousness, maintaining spontaneous ventilation. However, it should be remembered that maintaining the airway may be difficult because of changes in airway dynamics in infant patients, even though such an induction is performed at a low concentration of inhalation agent [8]. In very young patients, direct laryngoscopy is more preferred as the intubation method [11] because use of fiberoptic or rigid bronchoscopy is limited since special skills are required in case of emergency. In addition, tracheostomy is difficult when using only local anesthesia and anatomical landmarks are unclear. Hence, although difficulty during intubation was expected, we chose to use direct laryngoscopy. However, Bryan et al. [10] reported that endotracheal intubation was carried out using a fiberoptic bronchoscopy for a 3-week old infant with giant cystic lump, as they performed local anesthesia with $2 \%$ viscous lidocaine for the tongue and $2 \%$ lidocaine through epidural catheter for the glottis and airway, while maintaining spontaneous ventilation with midazolam and ketamine and inducing deep sedation. Additionally, it is necessary that a surgeon be ready during induction because immediate suction of the lump should be carried out when endotracheal intubation is difficult due to serious invasion into the oral cavity [7].

The second problem is airway maintenance during the operation. Since individual bronchial length varies (range, 5-9 $\mathrm{cm}$ ) in newborn and young infants, the proper positional range for endotracheal tube placement is narrow; consequently, the tube can be dislocated from the vocal cord or inserted into the bronchus even by simple flexion or extension of the cervical vertebra [12]. According to Todres et al. [13], endotracheal tube location could vary up to $28 \mathrm{~mm}$ depending on location of the cervical vertebra, as the median airway length was $57 \mathrm{~mm}$. Weiss et al. [14] also reported that the median bronchus length was 45.6 (range, $36.4-52.2 \mathrm{~mm}$ ) at the age of 0.4 , and location of the endotracheal tube tip could vary up to $10 \mathrm{~mm}$ if the cervical vertebra flexion was 30 degrees. In our case, considering that the operation position was hyperextension and right rotation of the cervical vertebra, the tube was fixed at a depth of $1 \mathrm{~cm}$ before the carina was found by auscultation. However, we overlooked the possible traction of larynx and airway by not only the operation position but also manipulation to secure visibility during the operation. Hence, it is assumed that the possibility of intraoperative extubation of the endotracheal tube or endobronchial intubation should be considered when extensive cervical manipulation of the newborn infant is expected. If necessary, the accurate airway length needs to be measured using a fiberoptic bronchoscopy after intubation and the safety zone should be secured.

The final problem is determination of postoperative extubation time. It is known that paralysis of the 7th, 11th and 12th cranial nerves occurs in $20 \%$ of patients undergoin cystic hygroma operation [6], and even damage of both phrenic nerves has been reported [15]. In our case, there was no problem in extubation on the second day postop, but later, temporary paralysis was found at the 7 th cranial nerve. Thus, extubation should be tried after understanding the degree of invasion of lumps by operation and the degree of nerve damage around the airway, depending on the size and invasion range of the lump.

In conclusion, for anesthesia of newborn or young infants undergoing giant lump removal surgery, the anesthetist needs to consider not only induction and endobroncheal intubation, but also intraoperative management of the endobronchial tube, anticipation of possible postoperative complications and determination of extubation time. 


\section{References}

1. Santillanes G, Gausche-Hill M. Pediatric airway management. Emerg Med Clin North Am 2008; 26: 961-75, ix.

2. Eckenhoff JE. Some anatomic considerations of the infant larynx influencing endotracheal anesthesia. Anesthesiology 1951; 12: 40110.

3. Brooks JE. Cystic hygroma of the neck. Laryngoscope 1973; 83: 11728.

4. Musone R, Bonafiglia R, Menditto A, Paccone M, Cassese E, Russo G, et al. Fetuses with cystic hygroma. A retrospective study. Panminerva Med 2000; 42: 39-43.

5. Kennedy TL. Cystic hygroma-lymphangioma: a rare and still unclear entity. Laryngoscope 1989; 99(10 Pt 2 Suppl 49): 1-10.

6. Gross E, Sichel JY. Congenital neck lesions. Surg Clin North Am 2006; 86: 383-92, ix.

7. Sharma S, Aminuldin AG, Azlan W. Cystic hygroma: anaesthetic considerations and review. Singapore Med J 1994; 35: 529-31.

8. Ishaq M, Minhas MR, Hamid M, Punjwani R. Management of compromised airway due to unusual presentation of cystic hygroma. J Pak Med Assoc 2006; 56: 135-7.
9. O'Donnell CP, Kamlin CO, Davis PG, Morley CJ. Endotracheal intubation attempts during neonatal resuscitation: success rates, duration, and adverse effects. Pediatrics 2006; 117: e16-21.

10. Bryan Y, Chwals W, Ovassapian A. Sedation and fiberoptic intubation of a neonate with a cystic hygroma. Acta Anaesthesiol Scand 2005; 49: 122-3.

11. Brooks P, Ree R, Rosen D, Ansermino M. Canadian pediatric anesthesiologists prefer inhalational anesthesia to manage difficult airways. Can J Anaesth 2005; 52: 285-90.

12. Griscom NT, Wohl ME. Dimensions of the growing trachea related to age and gender. AJR Am J Roentgenol 1986; 146: 233-7.

13. Todres ID, deBros F, Kramer SS, Moylan FM, Shannon DC. Endotracheal tube displacement in the newborn infant. J Pediatr 1976; 89: 126-7.

14. Weiss M, Gerber AC, Dullenkopf A. Appropriate placement of intubation depth marks in a new cuffed paediatric tracheal tube. Br J Anaesth 2005; 94: 80-7.

15. Koh SO, Oh HK. Bilateral diaphragmatic paralysis developed after explo-thoracotomy for removal of cystic hygroma. Korean J Anesthesiol 1987; 20: 561-4. 